DOI: $10.17516 / 1999-494 X-0225$

УДК 621.391, 621.373.8

\title{
Methods for Substantiating the Spatial Characteristics \\ of the Laser Beam of Telecommunication Systems \\ when Getting into Communication \\ with Airborne Control Objects
}

\author{
Vasily S. Kalinin a and Anton A. Koziratsky ${ }^{\mathbf{b} *}$ \\ ${ }^{a}$ Military Education and Research Centre of Military-Air Forces \\ "Military-Air Academy \\ Named After Professor N.E. Zhukovsky and Yu.A. Gagarin» \\ Voronezh, Russian Federation \\ ${ }^{b}$ Joint-Stock Company «Concern «Sozvezdie»» \\ Voronezh, Russian Federation
}

Received 22.11.2019, received in revised form 15.12.2019, accepted 21.01.2020

Abstract. Based on the method of functional conversion of random variables, a method has been developed for estimating the density of distribution of fluctuating radiation intensity of a transmitting laser at the input of the receiver, which allows to optimize the divergence angle when getting into communication, taking into account the accuracy of laser beam aiming and the fluctuation characteristics of laser radiation.

Keywords: accuracy of laser beam aiming, fluctuation of radiation intensity, optimization of divergence angle, getting into communication.

Citation: Kalinin V.S., Koziratsky A.A. Methods for substantiating the spatial characteristics of the laser beam of telecommunication systems when getting into communication with airborne control objects, J. Sib. Fed. Univ. Eng. \& Technol., 2020, 13(3), 338-349. DOI: 10.17516/1999-494X-0225

(C) Siberian Federal University. All rights reserved

This work is licensed under a Creative Commons Attribution-Non Commercial 4.0 International License (CC BY-NC 4.0).

* Corresponding author E-mail address: vasek888@list.ru 


\title{
Методика обоснования пространственных характеристик лазерного луча систем телекоммуникации \\ при вхождении в связь
}

с воздушными объектами управления

\author{
В.С. Калинин ${ }^{\mathbf{a}}$, А.А. Козирацкийб \\ ${ }^{a}$ Военныци учебно-научный изентр Военно-воздушных сил \\ «Военно-воздушная академия \\ имени профессора Н.Е. Жуковского и Ю.А. Гагарина» \\ Российская Федерация, Воронеж \\ ${ }^{\sigma}$ Акиионерное общество «Концерн «Созвездие»» \\ Российская Федерация, Воронеж
}

\begin{abstract}
Аннотация. На основе использования метода функционального преобразования случайных величин разработана методика оценки плотности распределения интенсивности флуктуирующего излучения передающего лазерного средства на входе приемника, позволяющая с учетом точности нацеливания лазерного луча и флуктуационных характеристик излучения лазера осуществлять оптимизацию угла расходимости при вхождении в связь.
\end{abstract}

Ключевые слова: точность нацеливания лазерного луча, флуктуация интенсивности излучения, оптимизация угла расходимости, вхождение в связь.

Цитирование: Калинин, В.С. Методика обоснования пространственных характеристик лазерного луча систем телекоммуникации при вхождении в связь с воздушными объектами управления / В.С. Калинин, А.А. Козирацкий // Журн. Сиб. федер. ун-та. Техника и технологии, 2020. 13(3). С. 338-349. DOI: 10.17516/1999-494X-0225

\section{Введение}

Увеличение дальности действия лазерных систем связи (при сохранении требуемой достоверности передачи сообщений) признано одной из значимых задач для реализации высокоскоростного защищенного информационного обмена с воздушными судами перспективных систем телекоммуникации. Основным фактором, определяющим увеличение дальности действия при постоянной мощности лазерного излучения передающего средства, является уменьшение угла расходимости лазерного луча, что, в свою очередь, позволит обеспечить требуемую достоверность передачи информации. Вместе с этим одна из трудностей - проблема нацеливания узкого луча, ширина которого соизмерима с величиной ошибок наведения и удержания его в требуемом направлении, особенно остро эта проблема проявляется на подвижных объектах [1- 3].

Кроме того, на практике генерация лазерного излучения сопровождается флуктуацией интенсивности, связанной спонтанным излучением [4], величина которого определяется типом лазера передающей системы и мерами, принятыми для повышения его стабильности $[5,6]$. При этом характеристики флуктуации зависят от параметра излучения $\xi$, изменяющегося в зависимости от приближения (или удаления) от порога генерации лазера.

Возникает актуальная задача оценки эффективности вхождения в связь с учетом точности нацеливания лазерного луча на приемную систему и флуктуирующих характеристик излучения лазера передающего средства.

$$
-339-
$$


Целью настоящей статьи является разработка методики оценки эффективности вхождения в связь, учитывающей точность нацеливания лазерного луча на приемник и флуктуационные характеристики излучения лазера, позволяющей увеличить дальности действия лазерных систем телекоммуникации (при сохранении требуемой достоверности передачи информации) за счет оптимизации угла расходимости передающего лазерного средства.

В качестве показателя эффективности приема лазерного излучения примем вероятность вхождения в связь $P_{c b}$.

Установление связи в системе телекоммуникации будет зависеть от выполнения двух совместных событий: события $A_{1}$, состоящего в том, что в результате нацеливания лазерный луч передающего средства будет ориентирован на апертуру приемника и «накроет»ее, и события $A_{2}$, состоящего в том, что интенсивность лазерного излучения на входе приемника превысит порог обнаружения при сохранении требуемой достоверности приема информации [7]. Необходимо заметить, что накрытие апертуры приемника будет зависеть от расходимости лазерного луча передающей системы, расстояния, на котором находится приемник, и ошибки нацеливания, и не будет зависеть от интенсивности лазерного излучения на входе приемника. В свою очередь, превышение порогового значения интенсивности лазерного излучения на входе приемника или обнаружение будет зависеть от интенсивности излучения на выходе лазера передающего средства, длины трассы распространения излучения (расстояния до приемника) и суммарного ослабления при ее прохождении в атмосфере. Таким образом, можно утверждать, что рассматриваемые события независимы, поэтому вероятность вхождения в связь определим выражением [8]

$$
P_{c B}=P\left(A_{1} \cdot A_{2}\right)=P\left(A_{1}\right) \cdot P\left(A_{2}\right),
$$

где $P\left(A_{1}\right)$ - вероятность события, состоящего в том, что в результате нацеливания лазерный луч передающего средства будет ориентирован на апертуру приемника и «накроет» ее (вероятность нацеливания); $P\left(A_{2}\right)$ - вероятность обнаружения сигнала в шумах.

\section{Нацеливание лазерного луча системой телекоммуникации}

Рассмотрим ситуацию, когда приемопередающие лазерные системы осуществили поиск и достигли ориентации в требуемом направлении (т.е. направлены друг на друга). При этом нацеливание лазерных лучей произведено с некоторой ошибкой.

На рис. 1 представлены поперечные сечения лазерного луча (пучка) двух приемопередающих систем «А» и «В» радиусом $a_{n}$ и $a_{n}^{\prime}$ в плоскостях формирующих оптических систем приемников, точки $Q$ и $Q^{\prime}$ определяют их центр, а точки $O$ и $O^{\prime}$ являются центрами апертур оптических систем приемников радиусом $a_{n p}$ и $a_{n p}{ }^{\prime}$. Расстояние между точками $Q\left(Q^{\prime}\right)$ и $O$ $\left(O^{\prime}\right)$, определяемое как $\rho_{\text {ou }}=a_{n}-a_{n p}$, характеризует ошибку нацеливания передатчика на приемник.

На практике в большинстве случаев ошибки нацеливания подчиняются закону Рэлея [8], поэтому

$$
w_{H}\left(\rho_{\text {ou }}\right)=\frac{\rho_{o u}}{\sigma_{l}^{2}} e^{-\frac{\rho_{o u}^{2}}{2 \sigma_{l}^{2}}},
$$




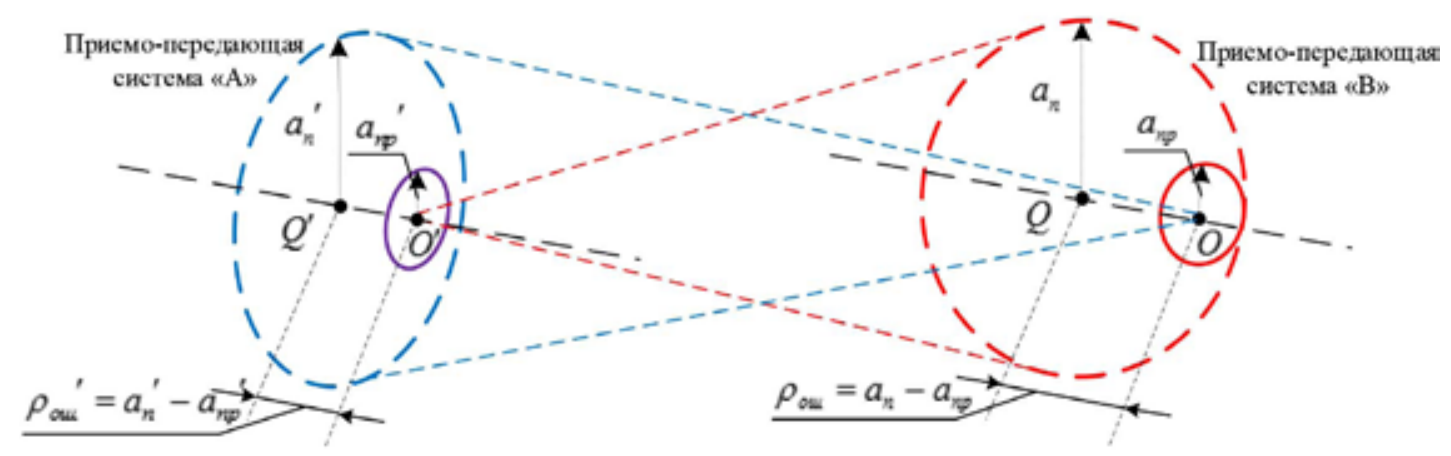

Рис. 1. Геометрия задачи

Fig. 1. Task geometry

где $\sigma_{l}-$ линейная среднеквадратическая ошибка нацеливания лазерного луча.

Для малоуглового приближения $\sigma_{l} \approx \sigma_{\alpha} L$, где $\sigma_{\alpha}$ - угловая среднеквадратическая ошибка нацеливания лазерного луча, $L$ - дальность между передающей и приемной системами.

В этом случае вероятность нацеливания лазерного луча на апертуру оптической системы приемника определим выражением

$$
P_{H}=P\left(A_{1}\right)=\int_{0}^{a_{n}-a_{n p}} w_{H}\left(\rho_{\text {ou }}\right) d \rho_{\text {ou }}=1-e^{-\frac{\left(a_{n}-a_{n p}\right)^{2}}{2 \sigma_{l}^{2}}} .
$$

На рис. 2 представлены графики зависимости вероятности нацеливания $P\left(A_{1}\right)$ от радиуса поперечного сечения луча $\alpha_{n}$ в плоскости апертуры приемника при различных значениях ошибки нацеливания $\sigma_{l}$, построенные с использованием выражения (3) для типового радиуса апертуры приемника $\alpha_{n p}=0,05$ м.

Анализ графиков, представленных на рис. 2, позволяет сделать вывод о том, что с увеличением расходимости лазерного луча вероятность нацеливания (накрытия апертуры приемника) увеличивается, однако с увеличением расходимости излучения плотность потока мощности

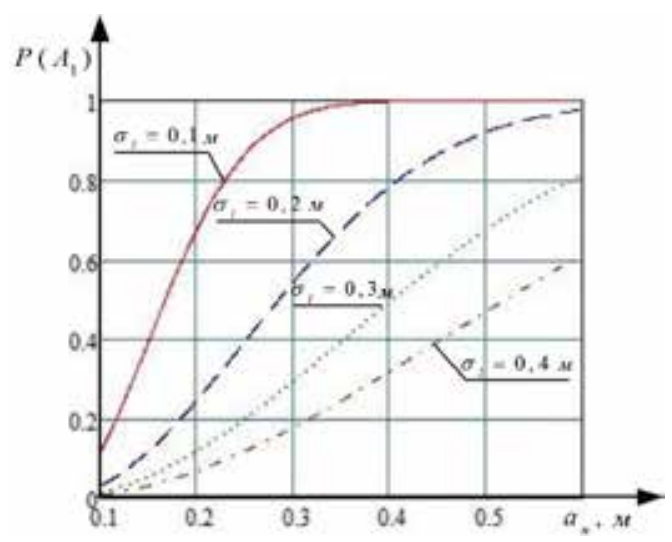

Рис. 2. Графики зависимости вероятности $P\left(A_{1}\right)$ от радиуса поперечного сечения луча $\alpha_{n}$

Fig. 2. The plot of the probability $P\left(A_{1}\right)$ of the radius of the cross section of the beam $\alpha_{n}$ 
Таблица 1. Результаты расчета оптимальных параметров и характеристик

Table 1. The results of the calculation of optimal parameters and characteristics

\begin{tabular}{|c|c|c|c|c|}
\hline $\begin{array}{c}\text { Значение угловой } \\
\text { среднеквадратической } \\
\text { ошибки наведения } \\
\sigma_{\alpha}, \text { рад }\end{array}$ & $\begin{array}{c}\text { Дальность } \\
L, \text { м }\end{array}$ & $\begin{array}{c}\text { Значение линейной } \\
\text { среднеквадратической } \\
\text { ошибки наведения } \\
\sigma_{l}, \mathrm{~m}\end{array}$ & $\begin{array}{c}\text { Оптимальный } \\
\text { радиус луча } a_{n}^{*}, \mathbf{м}\end{array}$ & $\begin{array}{c}\text { Угол расходимости } \\
\alpha_{p}^{*}, \text { рад }\end{array}$ \\
\hline \multirow{2}{*}{$2 \cdot 10^{-5}$} & $5 \cdot 10^{3}$ & 0,1 & 0,26 & $1,7 \cdot 10^{-4}$ \\
\cline { 2 - 5 } & $10 \cdot 10^{3}$ & 0,2 & 0,5 & $10^{-4}$ \\
\cline { 2 - 5 } & $15 \cdot 10^{3}$ & 0,3 & 0,7 & $9 \cdot 10^{-5}$ \\
\cline { 2 - 5 } & $20 \cdot 10^{3}$ & 0,4 & 0,9 & $0,9 \cdot 10^{-5}$ \\
\hline
\end{tabular}

излучения на входе приемника уменьшается, таким образом, приемная система не обеспечит прием информации или обеспечит с низкой достоверностью. На этих графиках также видно, что при известной угловой среднеквадратической ошибке нацеливания подсистемы наведения $\sigma_{\alpha}$ для различных дальностей от передающей до приемной системы $L$ возможно определить оптимальный (необходимый и достаточный) радиус поперечного сечения луча $\alpha_{n}$ и оптимальный угол расходимости лазерного луча $\alpha_{p}^{*}$, обеспечивающие вероятность нацеливания $P_{t} \approx 0,9$. Результаты расчетов приведены в табл. 1.

\section{Оценка распределения интенсивности лазерного излучения} на входе приемной системы

Пусть лазер передающего средства работает в режиме значительно выше порогового ( $\xi>>0)$. В этом случае, как показано в [4], плотность распределения интенсивности при генерации лазера имеет форму гауссовской плотности со средним значением $\bar{J}=\xi \sqrt{\pi} J_{\Pi \Gamma}$ :

$$
w\left(J_{u 3 \pi}\right)=\frac{1}{\pi J_{\Pi \Gamma}} \exp \left\{-\left(\frac{J_{u 3 \pi}-\xi \sqrt{\pi} J_{\Pi \Gamma}}{\sqrt{\pi} J_{\Pi \Gamma}}\right)^{2}\right\}, \quad J_{u 3,} \geq 0,
$$

где $J_{П Г}-$ средняя пороговая интенсивность генерации лазера; $\xi$ - параметр, изменяющийся в зависимости от режима работы лазера (значительно ниже порога генерации до нуля вблизи порога и до большого положительного значения), который, в свою очередь, зависит от значений средней пороговой интенсивности генерации лазера.

Интенсивность лазерного излучения в поперечном сечении луча в области апертуры приемной системы определим выражением:

$$
J_{n p}=J_{u 33} \frac{a_{0}^{2}}{a_{n}^{2}} e^{-\alpha_{\Sigma} L},
$$

где $\alpha_{0}$ - начальный радиус луча на выходе передающего средства; $\alpha_{\Sigma}-$ коэффициент суммарного ослабления потока излучения в атмосфере за счет рассеяния и поглощения $[9,10]$.

При этом необходимо отметить, что измеряемая с помощью, например, дальномера или спутниковой подсистемы GPS приемопередающих систем дальность $L$ является случайной величиной, так как определяется с ошибками.

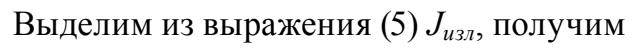




$$
J_{u з 3}\left(J_{n p}\right)=\frac{a_{n}^{2}}{a_{0}^{2}} e^{\alpha_{\Sigma} L} J_{n p} .
$$

Плотность распределения интенсивности лазерного излучения на входе приемной системы $w\left(J_{n p}\right)$ определим с использованием метода функционального преобразования случайных величин [8]:

$$
w\left(J_{n p}\right)=w\left(J_{\text {изл }}\left(J_{n p}\right)\right) \cdot\left|\frac{d J_{\text {изл }}}{d J_{n p}}\right| .
$$

Найдем производную выражения (6), получим

$$
\left|\frac{d J_{u 3 n}}{d J_{n p}}\right|=\frac{a_{n}^{2}}{a_{0}^{2}} e^{\alpha_{\Sigma} L} .
$$

Тогда на основе выражений (4), (6) и (8) распределение интенсивности флуктуирующего лазерного излучения на входе приемной системы запишем в следующем виде:

$$
w\left(J_{n p}\right)=\frac{1}{\pi J_{\Pi \Gamma}} \frac{a_{n}^{2}}{a_{0}^{2}} e^{\alpha_{\Sigma} L} \exp \left\{-\left(\frac{\frac{a_{n}^{2}}{a_{0}^{2}} e^{\alpha_{\Sigma} L} J_{n p}-\xi \sqrt{\pi} J_{\Pi \Gamma}}{\sqrt{\pi} J_{\Pi \Gamma}}\right)^{2}\right\} .
$$

На рис. 3 представлены графики зависимости распределения интенсивности флуктуирующего лазерного излучения $w\left(J_{n p}\right)$ от нормированной величины $k=J_{n p} / J_{П Г}$ при различных значениях радиуса поперечного сечения луча $\alpha_{n}$, построенные с использованием выражения (9). Характеристики передающего средства и типовые условия распространения лазерного излучения приведены в табл. 2.

Анализ графиков, представленных на рис. 3, позволяет сделать вывод о том, что изменение формы закона распределения существенным образом определяется расходимостью лазерного

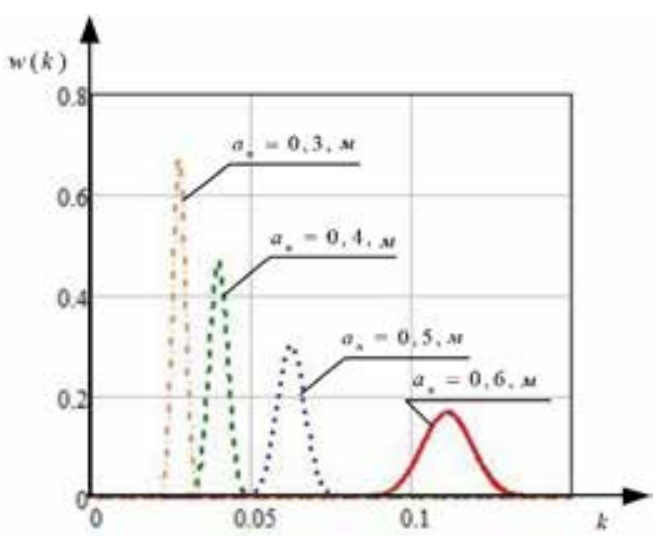

Рис. 3. Распределение интенсивности флуктуирующего лазерного излучения $w\left(J_{n p}\right)$ от нормированной величины $k=J_{n p} / J_{П Г}$ при различных значениях радиуса поперечного сечения луча $\alpha_{n}$

Fig. 3. The distribution of the intensity of fluctuating laser radiation from $w\left(J_{n p}\right)$ the normalized value $k=J_{n p} / J_{I I I}$ for various values of the radius of the beam cross section $\alpha_{n}$ 
Таблица 2. Характеристики передающего канала и условия распространения лазерного излучения

Table 2. Characteristics of the transmission medium and conditions for the propagation of laser radiation

\begin{tabular}{|l|c|c|c|c|c|c|}
\hline $\begin{array}{c}\text { Характеристика (параметр), } \\
\text { единица измерения }\end{array}$ & $J_{\Pi \Gamma}, B m / \mu^{2}$ & $D, \mathrm{M}$ & $\alpha_{0} \approx D / 4, \mathrm{M}$ & $\xi$ & $L, \mathrm{M}$ & $\alpha_{\Sigma}, \mathrm{M}$ \\
\hline Значение & 300 & 0,1 & 0,025 & 10 & $10^{4}$ & $10^{-5}$ \\
\hline
\end{tabular}

Таблица 3. Характеристики передающего канала и условия распространения лазерного излучения

Table 3. The results of the calculation of optimal parameters and characteristics

\begin{tabular}{|l|c|c|c|c|c|c|}
\hline $\begin{array}{c}\text { Характеристика (параметр), } \\
\text { единица измерения }\end{array}$ & $J_{\Pi \Gamma}, B m / M^{2}$ & $\theta$, рад & $a_{n}=\frac{1}{2} L \theta, \mathrm{M}$ & $\alpha_{0} \approx D / 4, \mathrm{~m}$ & $L, \mathrm{M}$ & $\alpha_{\Sigma}, \mathrm{M}$ \\
\hline Значение & 300 & $10^{-4}$ & 0,5 & 0,025 & $10^{4}$ & $10^{-5}$ \\
\hline
\end{tabular}

луча на входе приемной системы. При увеличении радиуса поперечного сечения луча $\alpha_{n}$ увеличивается дисперсия, максимальное значение плотности распределения при этом уменьшается, а среднее значение (математическое ожидание) увеличивается (распределение смещается вдоль оси абсцисс в область больших значений интенсивности флуктуирующего излучения).

На рис. 4 изображены графики зависимости распределения интенсивности флуктуиру-

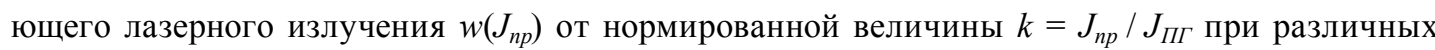
значениях параметра $\xi$, построенные с использованием выражения (9). Характеристики передающего канала и условия распространения приведены в табл. 3.

Проанализировав графики, выясняем, что с увеличением значения параметра $\xi$ увеличивается среднее значение (математическое ожидание) и распределения смещается вдоль оси абсцисс в область больших значений интенсивности флуктуирующего излучения, не изменяя своей формы.

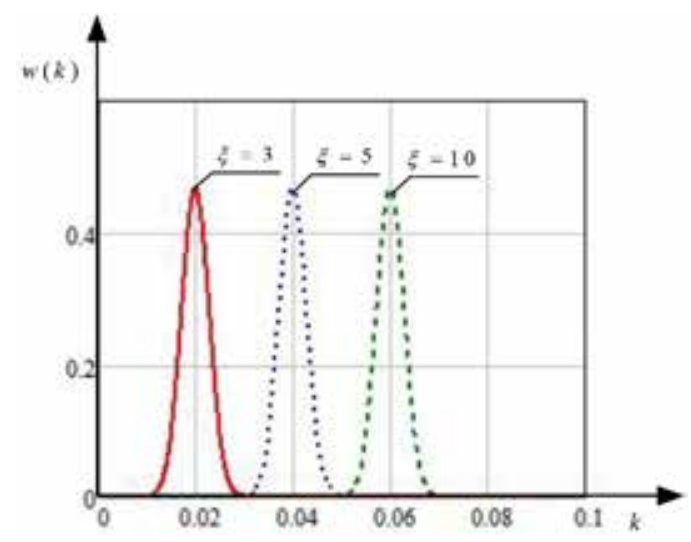

Рис. 4. Распределение интенсивности флуктуирующего лазерного излучения $w\left(J_{n p}\right)$ от нормированной величины $k=J_{n p} / J_{П Г}$ при различных параметрах $\xi$

Fig. 4. The distribution of the intensity of fluctuating laser radiation from $w\left(J_{n p}\right)$ the normalized value $k=J_{n p} / J_{I I I}$ for various parameters $\xi$ 
Таким образом, на основе использования метода функционального преобразования случайных величин получено выражение распределения интенсивности флуктуирующего лазерного излучения на входе приемной системы.

\section{Вероятность регистрации лазерного излучения средствами второго абонента}

Вероятность обнаружения сигнала приемной системой определим следующим выражением:

$$
P_{\text {обн }}=P\left(A_{2}\right)=\int_{J_{n . \text {. }}}^{\infty} w\left(J_{n p}\right) d J_{n p},
$$

где $J_{\text {n.o. }}$ - интенсивность порога обнаружения приемной системы.

Подставляя в выражение (10) закон распределения интенсивности лазерного излучения на входе приемной системы (9), получим:

$$
P_{\text {обн }}=1-\Phi\left[\sqrt{\frac{2}{\pi}}\left(\frac{a_{n}^{2}}{a_{0}^{2}} e^{\alpha_{\Sigma} L} \frac{J_{n . \text {.о. }}}{J_{\Pi Г}}-\sqrt{\pi} \xi\right)\right],
$$

где $\Phi(x)=\frac{1}{\sqrt{2 \pi}} \int_{0}^{x} e^{-\frac{t^{2}}{2}} d t-$ интеграл вероятности.

На рис. 5 представлены графики зависимости вероятности обнаружения $P\left(A_{2}\right)$ от радиуса поперечного сечения луча $\alpha_{n}$. Зависимости получены для значений интенсивности порога обнаружения $J_{\text {n.o. }}=100,150,200 \mathrm{BT} / \mathrm{M}^{2}$ с использованием выражения (11) и характеристик передающего средства, условий распространения лазерного излучения, приведенных в табл. 3.

Анализ графиков на рис. 5 позволяет сделать вывод о том, что при больших интенсивностях порога обнаружения приемной системы необходимо значительно уменьшить радиус поперечного сечения луча, следовательно, увеличить интенсивность мощности излучения, которая ограничена (постоянна). Одновременно уменьшение радиуса поперечного сечения луча в

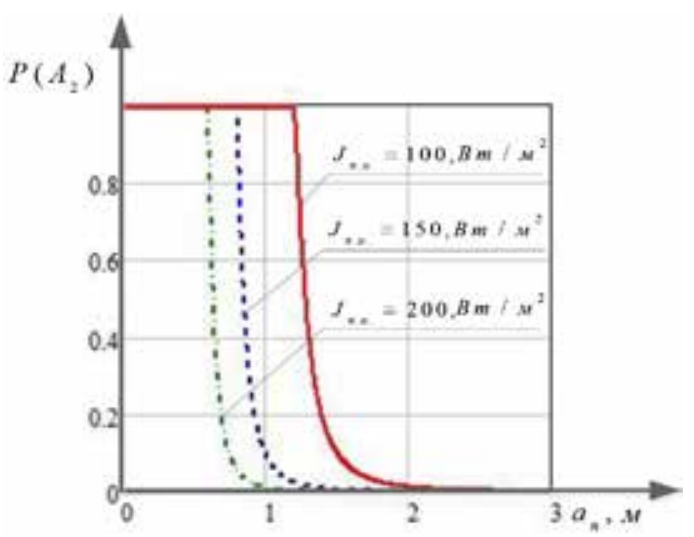

Рис. 5. График зависимости вероятности обнаружения $P\left(A_{2}\right)$ от радиуса поперечного сечения луча $\alpha_{n}$ при различных значениях интенсивности порога обнаружения $J_{\text {n.o. }}$

Fig. 5. The dependence of the probability $P\left(A_{2}\right)$ of detection on the radius $\alpha_{n}$ of the beam cross section for different values of the intensity of the detection threshold $J_{n . o}$. 
условиях существующих ошибок нацеливания может привести к неточной ориентации лазерного луча передающей системы на апертуру оптической системы приемника или «промаху».

\section{Исследование эффективности вхождения в связь.}

\section{Обоснование рациональных пространственных характеристик лазерного луча системы телекоммуникации}

Вероятность вхождения в связь определим в соответствии с выражением (1), подставляя в него полученные выражения вероятности нацеливания лазерного луча (3) на апертуру приемной системы и вероятности обнаружения (11), получим

$$
P_{c B}=\left(1-e^{-\frac{\left(a_{n}-a_{n p}\right)^{2}}{2 \sigma_{l}^{2}}}\right) \cdot\left[1-\Phi\left[\sqrt{\frac{2}{\pi}}\left(\frac{a_{n}^{2}}{a_{0}^{2}} e^{\alpha_{\Sigma} L} \frac{J_{n . o .}}{J_{\Pi \Gamma}}-\sqrt{\pi} \xi\right)\right] .\right.
$$

На рис. 6 представлены графики зависимости вероятности вхождения в связь $P_{c \varepsilon}\left(\alpha_{n}\right)$ от радиуса поперечного сечения луча $\alpha_{n}$ при различных значениях угловых среднеквадратических ошибках нацеливания $\sigma_{\alpha}$ на дальности $L=10^{4}$ м.

Как видно из представленных на рис. 6 зависимостей, с увеличением радиуса поперечного сечения луча $\alpha_{n}$ вероятность вхождения в связь увеличивается до определенного значения, что обусловливает увеличение вероятности нацеливания лазерного луча, а затем падает, что соответствует недостаточной интенсивности лазерного излучения для обнаружения на входе приемной системы. Поэтому можно сделать вывод о том, что существует оптимальное значение радиуса поперечного сечения луча $a_{n}^{*}$, обеспечивающего максимальное значение вероятности вхождения в связь, при этом с увеличением точности нацеливания (уменьшении $\sigma_{\alpha}$ ) вероятность вхождения в связь значительно увеличивается.

Для определения оптимального значения радиуса поперечного сечения луча исследуем выражение (12) на экстремум [11]. Найдем производную выражения (12) по параметру $\alpha_{n}$ и, приравнивая ее к нулю, получим:

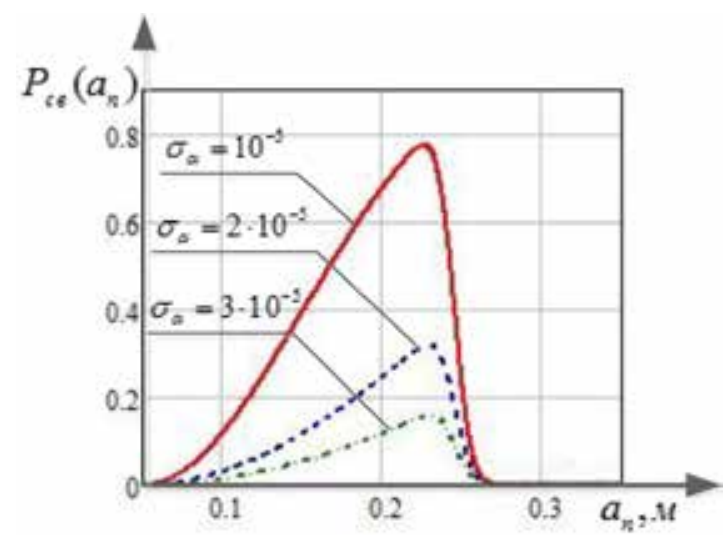

Рис. 6. График зависимости вероятности вхождения в связь $P_{c в}\left(\alpha_{n}\right)$ от радиуса поперечного сечения луча $\alpha_{n}$ при различных значениях угловой ошибки наведения $\sigma_{\alpha}$

Fig. 6. The dependence of the probability of joining $P_{c s}\left(\alpha_{n}\right)$ the relationship on the radius $\alpha_{n}$ of the beam cross section for various values of the angular pointing error $\sigma_{\alpha}$ 


$$
\left(1-e^{-\frac{\left(a_{n}-a_{n p^{2}}\right)^{2}}{2 \sigma_{l}^{2}}}\right) \cdot\left[1-\frac{1}{a_{n}}\left(J_{\Pi I}^{2}+1\right) \operatorname{erf}[x]+\frac{2 a_{n} J_{n .0} J_{\Pi I}}{\pi} \exp \left\{\alpha_{\Sigma} L-x^{2}\right\}\right]+\left(\frac{\left(a_{n}-a_{n p}\right)}{2 \sigma_{l}^{4}} e^{-\frac{\left(a_{n}-a_{n p}\right)^{2}}{2 \sigma_{l}^{2}}}\right) \cdot[1-\Phi[\sqrt{2} x]]=0,
$$

где $\operatorname{erf}[x]=\frac{2}{\sqrt{\pi}} \int_{0}^{x} e^{-x^{2}} d x-$ функция ошибок, $x=\frac{1}{\sqrt{\pi}}\left(\left(\frac{a_{n}}{a_{0}}\right)^{2} e^{\alpha_{\Sigma} L} \frac{J_{n . o .}}{J_{\Pi \Gamma}}-\sqrt{\pi} \xi\right)$.

Данное уравнение является трансцендентным, решить его точными методами не представляется возможным [11], поэтому решим его численным методом и при заданных в табл. 2 характеристиках передающего канала и условий распространения лазерного излучения для типового радиуса апертуры приемной системы $\alpha_{n p}=0,05$ м, интенсивности порога обнаружения $J_{\text {n.o. }}=50 \mathrm{BT} / \mathrm{M}^{2}$ и линейной среднеквадратической ошибке нацеливания $\sigma_{l}=0,2$ м получим корень уравнения $a_{n}^{*} \approx 0,24$ м.

Оптимальное значение угла расходимости излучения в общем случае $\alpha_{p}^{*}$ будет определяться выражением [12]

$$
\alpha_{p}^{*}=\frac{2}{L} a_{n}^{*} .
$$

На рис. 7 изображены графики зависимостей $P_{c b}(L)$ от дальности при оптимальном значении угла расходимости излучения $\alpha_{p}=\alpha_{p}^{*}$ и при $\alpha_{p}=$ const.

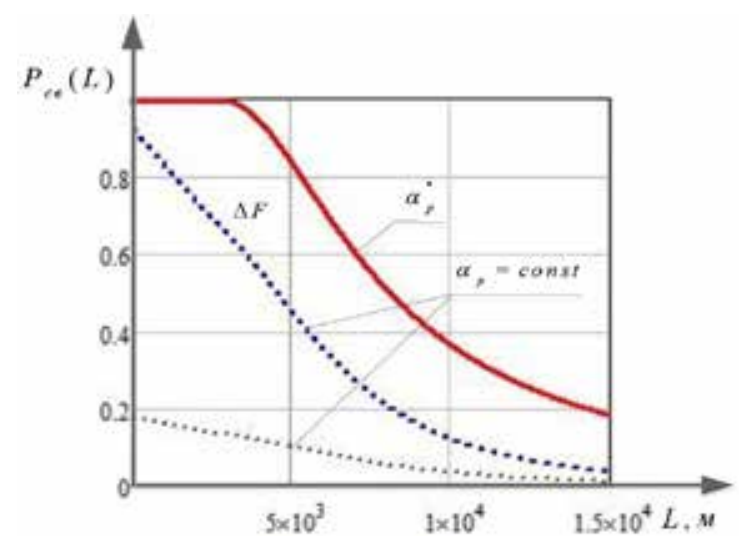

Рис. 7. График зависимости вероятности вхождения в связь $P_{c \varepsilon}(L)$ от дальности $L$

Fig. 7. The graph of the probability of joining the relationship $P_{c s}(L)$ from the range $L$

Как следует из данных графиков, формирование оптимального угла расходимости существенным образом повышает вероятность вхождения в связь. Так, на дальности 5 км за счет оптимизации угла расходимости вероятность $P_{c \varepsilon}$ повышается более чем в 1,8 раз и составляет величину 0,83 .

Итак, в процессе исследования установлено, что при вхождении в связь двух приемопередающих систем в заданных условиях и наличии ошибки нацеливания существуют оптимальные характеристики передающего средства, при формировании которых вероятность вхождения в связь значительно повышается. 


\section{Заключение}

Таким образом, на основе использования метода функционального преобразования случайных величин разработана методика оценки плотности распределения интенсивности флуктуирующего излучения лазера передающего средства на входе приемной системы, которая позволяет осуществлять оценку эффективности вхождения в связь с учетом точности нацеливания лазерного луча и флуктуационных характеристик излучения лазера передающего средства и обосновать основные требования к подсистеме наведения и формирования параметров лазерного луча бортовой телекоммуникационной системы.

\section{Список литературы / References}

[1] Шереметьев А.Г. Статическая теория лазерной связи, М.: Связь, 1971, 264 с. [Sheremetyev A.G. Static theory of laser communication, Moscow: Communication, 1971, 264 p. (in Russian)].

[2] Козирацкий А.Ю., Гревцев А.И., Капитанов В.В., Козирацкий А.А. Способ определения угловых координат объектов оптико-электронными средствами на основе пространственновременной обработки. Журнал Сибирского федерального университета. Техника и технологии, 2017, 2, 230-234 [Koziratsky A.Ju., Grevtsev A.I., Kapitanov V.V., Koziratsky A.A. The Method of Determining the Angular Coordinates of Objects by Electro-Optical Means on the Basis of SpaceTime Processing, Jornal Siberian Federal University, Engineering and Technologies, 2017, 2, 230 234 (in Russian)].

[3] Бутузов В.В., Козирацкий Ю.Л., Прохоров Д.В., Хильченко Р.Г. Оценка эффективности лазерной системы управления направлением разлета поражающих элементов снаряда при поражении воздушных целей. Журнал Сибирского федерального университета. Техника и технологии, 2019, 3, 266-274 [Butuzov V.V., Koziratsky Ju.L., Prohorov D.V., Hilchenko R.G. Evaluation of the Effectiveness of Laser System Control the Direction of Scattering Damaging Elements of the Projectile with the Defeat of Air Targets, Jornal Siberian Federal University, Engineering and Technologies, 2019, 3, 266-274 (in Russian)].

[4] Звелто О. Принципы лазеров, М.: Мир, 1990, 558 c. [Zvelto O. Principles of Lasers, Moscow: Mir, 1990, 555 p. (in Italy)].

[5] Гудмен Дж.В. Статическая оптика, М.: Мир, 1988, 527 с. [Goodman J.V. Static Optics, Moscow: Mir, 1988, 572 p. (in USA)].

[6] Козирацкий Ю.Л., Алабовский А.В. Аналоговое моделирование лазера с пассивной модуляцией добротности при жестком возбуждении генерации. Известия ВУЗов. Радиофизика, 1988, 10, 1264-1266 [Koziratsky Yu.L., Alabovsky A.V. Analog simulation of a laser with passive Q switching under hard excitation of generation. News of Universities. Radiophysics, 1988, 10, 1264 1266 (in Russian)].

[7] Козирацкий Ю.Л., Меркулов Р.Е., Кох Н.С. Обоснование принципов построения малогабаритной бортовой станции формирования упреждающих помех лазерным системам управления огнем зенитно-артиллерийского комплекса. Журнал Сибирского федерального университета. Техника и технологии, 2018, 1, 6-15 [Koziratsky Yu.L, Merkulov R.E., Koch N.S. Substantiation of Principles of Construction of Small-Sized Onboard Station of Formation of Anticipatory Hindrances to Laser Control Systems of Fire of an Antiaircraft- 
Artillery Complex, Jornal Siberian Federal University, Engineering and Technologies, 2018, 1, $6-15$ (in Russian)]

[8] Вентцель Е.С. Теория вероятностей. М.: Наука, 1969, 576 с. [Ventzel E.S. Probability Theory, Moscow: Nauka, 1969, 576 p. (in Russian)].

[9] Литвиненко О.Н. Основы радиооптики. Киев: Техніка, 1974, 208 с. [Litvinenko O.N. Fundamentals of Radio Optics, Kiev: Technics, 1974, 208 p. (in Russian)].

[10] Малашин М.С., Каминский Р.П., Малашин М.С., Борисов Ю.Б. Основы проектирования лазерных локационных систем. М.: Высшая школа, 1983, 206 с. [Malashin M.S., Kaminsky R.P., Malashin M.S., Borisov Yu.B. Fundamentals of designing laser location systems, Moscow: Higher school, 1983, 206 p. (in Russian)]

[11] Корн Г., Корн Т. Справочник по математике для научных работников и инженеров. М.: Наука, 1974, 832 с. [Korn G., Korn T. Handbook of mathematics for scientists and engineers, Moscow: Nauka, 1974, 832 p. (in Russian)].

[12] Козирацкий Ю.Л. Оптимизация угла расходимости излучения лазерной локационной системы. Радиотехника, 1994, 3, 6-10 [Koziratsky Yu.L. Optimization of the divergence angle of radiation of a laser location system, Radio Engineering, 1994, 3, 6-10 (in Russian)]. 